\title{
Bacterial profiling of head lice isolated from the Orang Asli: A first report in Malaysia
}

\author{
Abd Majid, M.A. ${ }^{1}$, Khoo, J.-J. ${ }^{1}$, Lim, F.-S. ${ }^{1}$, Khor, C.-S. ${ }^{1}$, Loong, S.-K. ${ }^{1}$, Low, V.-L. ${ }^{1}$, Lee, H.-Y. ${ }^{*}$ \\ and AbuBakar, S.1,2 \\ ${ }^{1}$ Tropical Infectious Diseases Research and Education Centre (TIDREC), University of Malaya, \\ 50603 Kuala Lumpur, Malaysia \\ ${ }^{2}$ Department of Medical Microbiology, Faculty of Medicine, University of Malaya, 50603 Kuala Lumpur, \\ Malaysia \\ *Corresponding author e-mail: leehaiyen@um.edu.my \\ Received 6 March 2020; received in revised form 22 July 2020; accepted 22 July 2020
}

\begin{abstract}
This study was carried out to determine from bacterial profiling to the bacterial profiles of head lice among the Orang Asli communities. The head lice were collected from Orang Asli community volunteers. The surface sterilized head lice pools were subjected to genomic DNA extraction while next generation sequencing of the 16S rRNA gene was performed using the Illumina MiSeq platform. Six female and three male head lice identified as Pediculus humanus capitis were collected. A total of 111368 number of NGS sequencing reads were recorded while another 223 bacterial taxa sequences were obtained. Symbiotic bacteria showed the highest number of reads, with Arsenophonus and Rhodococcus sequences being the most abundant genera in the female and male samples, respectively. The female head lice contained a more distinct microbial diversity. Amongst the pathogenic bacterial species sequences noted were the methicillin-resistant Staphylococcus aureus, Streptobacillus moniliformis, Haemophilus influenzae, Bordetella pertussis and Acinetobacter baumannii. The 16S rRNA genome sequencing revealed a number of rare and pathogenic bacterial species within the head lice of the Orang Asli. The socio-economic practices of the community which involved forest foraging and hunting, and their poor living conditions potentially facilitated the transmission of zoonotic bacterial pathogens, including those found within the head lice. Hence, there is the possibility that the head lice could serve as vectors for the transmission of pathogenic bacteria. This study highlighted the diverse microbial community found within the head lice's gut of the Orang Asli, with the detection of multiple rare and pathogenic bacteria capable of causing severe infections.
\end{abstract}

\section{INTRODUCTION}

Globally, ectoparasitic infestations caused by head lice are an annoyance (Lesshafft $e t$ $a l ., 2013)$, especially to pre-school children and the poor communities of the developing world (Feldmeier, 2014). Humans can be infested by two lice species, a.) the human louse, Pediculus humanus, represented by two sub-species, the head louse (Pediculus humanus capitis) and the body louse (Pediculus humanus humanus); and b.) the pubic louse (Pthirus pubis) (Mumcuoglu, 2008).
The head louse is the most common and it is highly distributed with a prevalence of up to $61 \%$ throughout the world (Falagas et $a l ., 2008)$. The head louse is found on the human scalp and it feeds by sucking blood repeatedly. Female louse cements its eggs (nits) firmly to the base of the hair shaft after 5 to 9 days. Head louse infestation can cause intense scalp itchiness, dermatitis, and lymphadenitis due to the repeated inoculation of the louse saliva (Saghafipour et al., 2017). The prolonged and chronic infestation by the head lice may in rare cases also cause anaemia, which is manifested by fatigue, 
sleepiness as well as poor cognitive and learning function, especially among children (Bachok et al., 2006).

To date, it is still unclear if the head lice can act as vectors of human pathogens. However, previous studies have reported the detection of Bartonella quintana DNA in head lice collected from Nepalese children (Sasaki et al., 2006), homeless individuals from the USA (Bonilla et al., 2009), Ethiopian patients (Angelakis et al., 2011a), as well as nits collected from a homeless man in France (Angelakis et al., 2011b). Previous studies on the prevalence of head lice in Malaysia focused on school-going children (Sinniah et al., 1981; Bachok et al., 2006; Muhammad Zayyid et al., 2010), suggested an upsurge in the prevalence of head lice infestations among Malaysian children from $10.7 \%$ in 1981 (Sinniah et al., 1981) to $35.0 \%$ in 2006 (Bachok et al., 2006) and 49.0\% in 2010 (Muhammad Zayyid et al., 2010). To make matters worse, the resistance of head lice to pediculicides has been reported and is on the rise (Frankowski et al., 2002). Most of the indigenous people or the Orang Asli of Malaysia that live within the forest fringe areas still follow the subsistence way of life, although some prefer to work in oil palm or rubber plantations (Masron et al., 2013; Khor et al., 2018). Their socio-economic practices and dependence on the forest expose them to the risk of contracting infectious diseases. These reasons are attributed to the fact that over $60 \%$ of the Orang Asli population in Kuala Milot to be infested with head lice (Vythilingam et al., 1998). Aside from that and to the best of our knowledge, there has been no other study to look at the prevalence of head lice among the Orang Asli people.

Here, we sought to perform bacterial profiling of head lice isolated from the Orang Asli in Malaysia. The aim of the study was to identify potentially pathogenic bacterial species infecting this economically challenged population.

\section{MATERIALS AND METHODS}

Ethics, consent, and permission for sample collection

This study was conducted in April 2017 in an Orang Asli settlement $\left(101.917726^{\circ} \mathrm{E}\right.$, $3.143844^{\circ} \mathrm{N}$ ), located in the state of Selangor, Malaysia. Sample collections were performed with approval from the Ethics Committee of the University Malaya Medical Centre, Malaysia (MREC: 20161115-4602) and the Department of Orang Asli Development, Malaysia (JAKOA). Prior to sample collection, questionnaires and written consent forms were distributed to each participant and sampling was only performed after receiving consent. Consent was also obtained from respective parents or guardians on behalf of their children who are under the age of 18 .

A fine-tooth comb was used to remove the head lice from the volunteer's hair. At the time of sampling, three female volunteers from 30 participants were afflicted with the head lice. The samples were kept in liquid nitrogen and transported to the Tropical Infectious Diseases Research \& Education Centre (TIDREC), University of Malaya, followed by storage in $-80^{\circ} \mathrm{C}$.

\section{Identification of head lice samples}

The collected head lice were observed under the microscope to identify the species and further classify the life stages based on the published taxonomic keys for Pediculus lice (Bonilla et al., 2013).

\section{DNA extraction}

The head lice were pooled according to their gender and surface sterilized strictly following the established protocols (Lim et al., 2017). Briefly, pooled samples were pulverized and powdered in liquid nitrogen using chilled mortar and pestle. The powdered samples were then suspended with sterile phosphate-buffered saline followed by 
DNA extraction using the NucleoSpin Tissue kit (Macherey-Nagel, Duren, Germany). The extracted genomic DNA from the male and female head lice pools were sent to a thirdparty service provider (Biolution Resources, Selangor, Malaysia) for metagenomics analysis using the Illumina MiSeq platform.

\section{Data and sequence analysis}

The generated raw reads were stored in fastq format, followed by demultiplexing and quality-filtering using QIIME (version 1.9.1). The paired-reads were merged into a single read and subjected to operational taxonomic unit (OTU) clustering, with 97\% similarity cut off using UPARSE (http://drive5.com/ uparse). In parallel, chimeric sequences were identified and removed using UCHIME (http://www.drive5.com/uchime/). The taxonomy of each sequence was analysed by RDP Classifier (http://rdp.cme.msu.edu/) against the SILVA 16S rRNA database (http:// www.arb-silva.de) using the confidence threshold of 0.7 . The rarefaction curves were plotted to assess species richness in the respective samples using mothur (https:// www.mothur.org/). The bacterial community composition in the samples was computed using $\mathrm{R}$ programming language (http://www.rproject.org/).

\section{Phylogenetic tree analysis of selected bacterial taxa}

Selected bacterial genera or species were compared for similarity with other pathogenic strains and their phylogenetic relationships inferred using the neighbourjoining method implemented in MEGA (version 6.0). The phylogenetic tree was tested using the Kimura-2 parameter algorithm with 1000 bootstrap replicates.

\section{RESULTS}

\section{Head lice samples}

Nine adult head lice were identified as Pediculus humanus capitis, comprising of six females and three males. They were collected from three female children from Orang Asli volunteers from a total of 50 volunteers (adults and children).

\section{S rRNA sequencing results}

The amplicons for the bacterial 16S rRNA gene of the pooled male sample yielded 53232 number of reads, while the pooled female sample yielded 58136 number of reads for downstream analysis. A total of 53655 (around 421-440bp) and 59259 (around 441-460bp) valid sequences were generated after assembly and trimming using the Trimmomatic and FLASH softwares (Majorbio, China). The samples were rarefied for OTU clustering and taxonomic assignment for the subsequent bacterial diversity analysis.

\section{Bacterial community detected in head louse samples}

The rarefaction curves for the male pool approached saturation at 53200 reads while the female reported 57800 reads, detecting a total of 223 taxa. At the class level, six major classes were observed, namely, Actinobacteria (31/223, taxa/total taxa), Alphaproteobacteria (20/223), Bacteroidia (26/223), Bacilli (16/223), Betaproteobacteria (16/223), and Gammaproteobacteria (21/223). Only 14 bacterial taxa were most represented $(\geq 0.5 \%)$ for both pools combined (93.96\%), in which 12 out of 14 bacterial taxa were assigned to the genus level (90.59\%) (Table 1). A total of 145 bacterial taxa were found in the female pool while 163 taxa were identified in the male pool.

Out of the total 111368 number of reads, Rhodococcus was the most abundant genus found in both the male and female pools (31.85\%), followed by Arsenophonus (20.91\%), Staphylococcus (13.96\%), Microbacterium (9.75\%), Haemophilus (6.59\%), Escherichia-Shigella (1.94\%), Streptococcus (1.65\%), Pseudomonas (1.37\%), Bordetella (0.76\%), Propionibacterium (0.72\%), Achromobacter (0.56\%), and Bacteroides (0.53\%) (Table 1). Arsenophonus (37.35\%) and Rhodococcus (36.74\%) were the most dominant genera identified in the female and male pools, respectively (Figure 1). Meanwhile, $0.08 \%$ and $0.47 \%$ of the reads in the respective female and male pools, were not assigned to any genus. 
Table 1. Assigned taxa with abundance $>0.5 \%$ of total population in head lice samples

Assigned taxa

Abundance

d__Bacteria; p__Actinobacteria; c__Actinobacteria; o___Corynebacteriales;

31.85

f__Nocardiaceae; g__Rhodococcus

d__Bacteria; p__Proteobacteria; c__Gammaproteobacteria; o__Enterobacteriales;

20.91

f_Enterobacteriaceae; g__Arsenophonus

d__Bacteria; p__Firmicutes; c__Bacilli; o__Bacillales; f__Staphylococcaceae;

13.96

g__Staphylococcus

d__Bacteria; p__Actinobacteria; c__Actinobacteria; o__Micrococcales;

f__Microbacteriaceae; g___Microbacterium

d__Bacteria; p__Proteobacteria; c__Gammaproteobacteria; o__Pasteurellales;

f__Pasteurellaceae; g__Haemophilus

d__Bacteria; p__Firmicutes; c__Clostridia; o__ Clostridiales; f__Other; g__Other 2.23

d__Bacteria; p__Proteobacteria; c__Gammaproteobacteria; o__Enterobacteriales;

f__Enterobacteriaceae; g__Escherichia-Shigella

d__Bacteria; p__Firmicutes; c__Bacilli; o__Lactobacillales; f__Streptococcaceae;

1.65

g__Streptococcus

d__Bacteria; p__Proteobacteria; c__Gammaproteobacteria; o__Pseudomonadales;

1.37

f__Pseudomonadaceae; g__Pseudomonas

d__Bacteria; p__Proteobacteria; c__Alphaproteobacteria; o__Other; f__Other; g___Other 1.14

d__Bacteria; p__Proteobacteria; c__Betaproteobacteria; o__Burkholderiales; 0.76

f__Alcaligenaceae; g__Bordetella

d__Bacteria; p__Actinobacteria; c__Actinobacteria; o__Propionibacteriales; 0.72

f__Propionibacteriaceae; g__Propionibacterium

d__Bacteria; p__Proteobacteria; c__Betaproteobacteria; o__Burkholderiales;

f__Alcaligenaceae; g__Achromobacter

d__Bacteria; p__Bacteroidetes; c__Bacteroidia; o__Bacteroidales; f__Bacteroidaceae; $\quad 0.53$

g__Bacteroides

*d: domain; p: phylum; c: class; o: order; f: family; g: genus; >: greater than; \%: percentage.

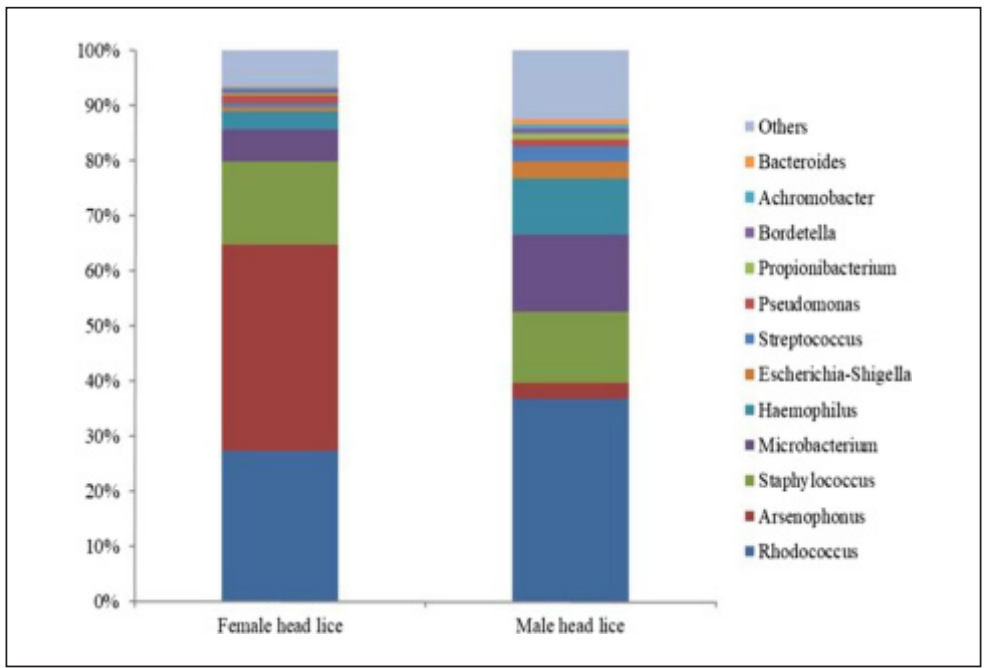

Figure 1. Relative abundance of bacterial populations at the genus level in head lice samples. 
The detected bacterial taxa in the head lice samples were broadly separated into three groups comprising of the endosymbionts (Candidatus Riesia pediculicola and Rhodococcus), the medically-important/ potentially pathogenic bacteria (Streptococcus salivarius, S. anginosus, Staphylococcus aureus, S. sciuri, Streptobacillus moniliformis, Propionibacterium acnes, Citrobacter koseri, Bergeyella zoohelcum, Ralstonia pickettii, Bordetella pertussis, Empedobacter brevis, Parabacteroids distasonis, Haemophilus influenzae and Acinetobacter baumannii) and the environmental bacteria (Bacillus, Methylobacterium, and Sphingomonas) (Table 2). $S$. aureus was the most prevalent bacterial species observed in both female and male pools, and potentially pathogenic bacteria was more abundantly found in the male lice.

\section{Phylogenetic tree of selected pathogenic bacterial species}

The 16S rRNA sequence analysis between the medically-important/potentially pathogenic bacterial species found in this study and other clinical isolates obtained from the GenBank was shown in Figure 2. From the phylogenetic tree, OTU20 and OTU169 were clustered into the streptococci clade together with $S$. anginosus (NR041722) and $S$. salivarius (AF459433), while the staphylococci clade gathered OTU41, OTU52, $S$. aureus (AM980864) and S. sciuri (AB662960). OTU64, OTU 125 and OTU143 were grouped with Bergeyella sp. (AY289204 and AY398696), E. brevis (NR042471) and $P$. distasonis (AB238922 and AB238924), possibly reflecting their origin from the bacterial phylum Bacteroidetes. OTU67 and OTU111 were clustered together with $B$.

Table 2. Relative abundance of different types of bacteria isolated from female and male head lice

\begin{tabular}{|c|c|c|c|}
\hline \multirow{2}{*}{ OTU ID } & \multirow{2}{*}{ Bacterial taxon } & \multicolumn{2}{|c|}{ Relative abundance (\%) } \\
\hline & & Female & Male \\
\hline \multicolumn{4}{|c|}{ Endosymbiont bacteria } \\
\hline OTU117 & Candidatus Riesia pediculicola & 37.3 & 2.96 \\
\hline OTU173 & Rhodococcus sp. & 30 & 36 \\
\hline \multicolumn{4}{|c|}{ Medically-important/pathogenic bacteria } \\
\hline OTU20 & Streptococcus salivarius & 0.12 & 0.2 \\
\hline OTU41 & Staphylococcus aureus MRSA & 15 & 12.77 \\
\hline OTU42 & Streptobacillus moniliformis & 0 & 0.39 \\
\hline OTU52 & Staphylococcus sciuri subsp. sciuri & 0.05 & 0 \\
\hline OTU53 & Propionibacterium acnes & 0.33 & 1.12 \\
\hline OTU58 & Citrobacter koseri & 0.007 & 0.22 \\
\hline OTU64 & Bergeyella zoohelcum & 0 & 0.33 \\
\hline OTU67 & Ralstonia pickettii & 0.03 & 0 \\
\hline OTU111 & Bordetella pertussis & 0.64 & 0.9 \\
\hline OTU125 & Empedobacter brevis & 0.11 & 0 \\
\hline OTU143 & Parabacteroides distasonis & 0.03 & 0 \\
\hline OTU161 & Haemophilus influenzae & 3.32 & 10.16 \\
\hline OTU167 & Acinetobacter baumannii & 0.06 & 0.008 \\
\hline OTU169 & Streptococcus anginosus subsp. anginosus & 0 & 0.05 \\
\hline \multicolumn{4}{|c|}{ Environmental bacteria } \\
\hline OTU127 & Bacillus sp. & 0 & 0.024 \\
\hline OTU5 & Methylobacterium sp. & 0.366 & 0 \\
\hline OTU27 & Sphingomonas echinoides & 0.179 & 0.036 \\
\hline OTU103 & Sphingomonas kaistensis & 0 & 0.085 \\
\hline OTU153 & Sphingomonas aerolata & 0.05 & 0 \\
\hline
\end{tabular}

*OTU: Operational Taxonomic Unit; \%: percentage. 


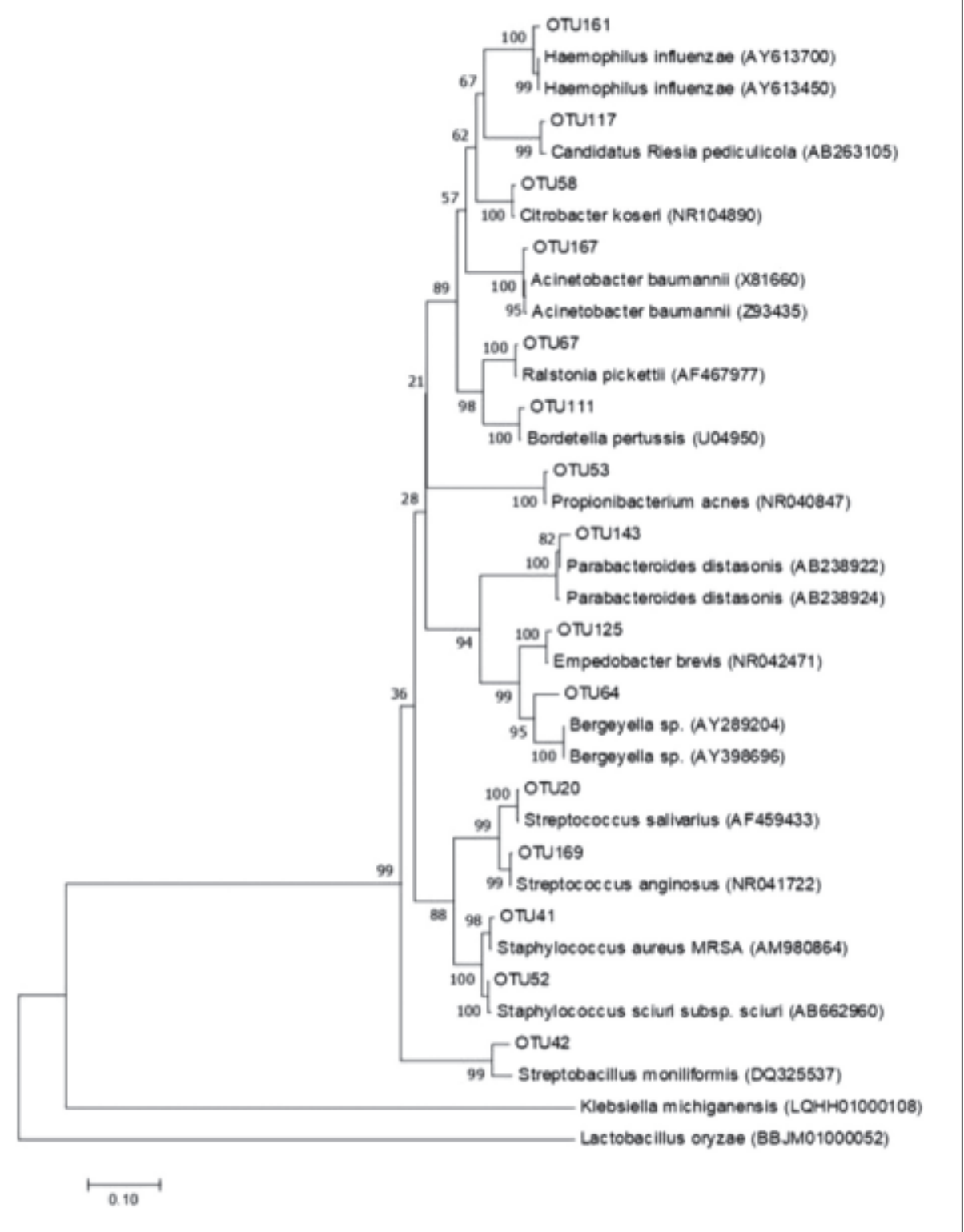

Figure 2. Phylogenetic tree derived from 16S rRNA bacteria sequences isolated from head lice in the present study compared to other bacterial isolates available from GenBank. The tree was calculated using neighbor-joining method. The numbers on branch points depict the values of 1000 bootstrap replicates. GenBank accession numbers for reference sequences are indicated at the end of each species designations.

pertussis (U04950) and $R$. pickettii (AF467977), suggesting that they belonged to the class $\beta$-proteobacteria. OTU58, OTU161 and OTU167 likely belonged to the class $\gamma$-proteobacteria as they were grouped with $A$. baumannii (X81660 and
Z93435), C. koseri (NR104890) and $H$. influenzae (AY613700 and AY13450). OTU117 and OTU53 were clustered respectively with $C$. $R$. pediculicola (AB263105) and P. acnes (NR040847). 


\section{DISCUSSION}

The indigenous communities living in forest-fringe areas, located away from modern healthcare infrastructures are often economically marginalised (Masron $e t a l$., 2013; Khor et al., 2018). Numerous initiatives to improve the living standards of the Orang Asli communities had been carried out by the Malaysian government over the years, including the continuous education and awareness campaigns on hygiene practices (Chandren et al., 2015). Despite all these efforts, head lice infestation remains a problem. Vythilingam et al. (1998) reported the only head lice prevalence study in an Orang Asli village in Pahang, Malaysia, however, they did not document the diversity of potentially pathogenic bacterial species found within the head lice, hence, could not ascertain if head lice could serve as vector for transmission of pathogens.

Arthropods are colonised by numerous microorganisms, amongst them bacteria that share symbiotic relationships important for the host nutrition, growth, and pathogen defence (Chen et al., 2016). Head lice infestation among humans is commonly associated with people from the lower socioeconomic groups (Falagas et al., 2008) with children being frequently affected (Meister \& Ochsendorf, 2016). Similar incidences had also been monitored among the Orang Asli children in Perak with 46.3\% being infested with head lice. Based on the report, girls (74.7\%) showed a higher prevalence compared to boys (13.2\%) (Soe et al., 2018).

The 16S gene was used for the investigation of bacterial diversity in the head lice because it has been successfully utilised for the detection of rare and pathogenic bacterial species (Schrottner et al., 2016). Given that the head lice have a restricted hematophagous diet (Rodríguez et al., 2011), they could host endosymbionts to provide them with vitamins and other nutrients essential for development and growth. It was not surprising, therefore, to find relatively high numbers of the genera Rhodococcus (Rodríguez et al., 2011), Arsenophonus (Šochová et al., 2017), and C.
R. pediculicola (Allen et al., 2007), which are known head lice endosymbionts (Tables 1 and 2). The socio-economic status and daily routine activities of the Orang Asli which involves forest foraging, very likely exposes them to ubiquitous environmental bacteria such as the Bacillus sp. (Diamoande et al., 2015), Methylobacterium sp. (Gallego et al., 2005), and Sphingomonas sp. (Leys et al., 2004), encountered in this study, albeit at very low relative abundances (Table 2).

Acinetobacter sp. stood out as the most commonly detected pathogenic bacteria in the human head lice, besides B. quintana and $B$. recurrentis (Mana et al., 2017). However, this study revealed a higher presence of other pathogenic bacteria (in terms of relative abundance) that had not been widely reported to be associated with human head lice. Our findings detected more female head lice infestations, similar to a previous study (Perotti et al., 2004). Nonetheless, pathogenic bacterial species was found relatively higher in the male (26.148\%) compared to female pools (19.697\%). Although several questions arise on the gaps of pathogenic density between male and female head lice, both sexes could potentially harbour a number of pathogens detrimental to human health.

Staphylococci represented the highest medically-important bacteria genera detected in the head lice samples (Table 1 and Figure 1), particularly methicillinresistant $S$. aureus (MRSA). Abrasions of the scalp due to head lice infestation could lead to soft tissue infections by the MRSA (Sam et al., 2008). The acquisition of $S$. sciuri by head lice could possibly reflect transmission from animals to the Orang Asli as this bacterium is usually an animal skin colonizer (Beims et al., 2016). Likewise, $S$. moniliformis (Table 2) and B. zoohelcum (Figure 2) were most likely to have originated from the wild rats (Suzuki et al., 2017) and pets (Chen et al., 2017), respectively. It is reasonable to suggest that these transmissions are due to frequent contacts with domesticated or wild animals that are consistent with the socio-economic practices of the Orang Asli. 
The next highest relative microbial abundance found in the head lice samples belonged to the genera Microbacterium and Haemophilus (Table 1 and Figure 1). Members of both genera have been implicated in clinical cases with $M$. paraoxydans causing dialysis-related peritonitis (Choi et al., 2017) and Haemophilus influenzae, the leading cause of meningitis, epiglottitis, and pneumonia in children under five years old (Monge et al., 2018). This pathogen is covered in the national vaccination program. Bordetella pertussis, the agent of whooping cough, found in the studied head lice samples, is also covered by the Malaysian vaccination program (Wong et al., 2008). These examples illustrate the risk of infectious diseases transmission to the Orang Asli, as the pathogenic bacteria exist in relatively high abundance in the collected head lice.

Apart from that, sequencing of the collected head lice detected a number of opportunistic pathogens, supposedly existing as commensal bacteria in many mammals. $P$. acnes (Figure 2), primarily found on the mammalian skin, has been associated with invasive and nosocomial infections (Achermann et al., 2014). The head lice could have acquired this bacterium from the scalp of the Orang Asli. Similarly, S. salivarius (Delorme et al., 2011), S. anginosus (Erdem et al., 2015), C. koseri (Dzeing-Ella et al., 2009), R. pickettii (Stelzmueller et al., 2006), and $P$. distasonis (Kierzkowska et al., 2017) (Figure 2) are commensals of the oral cavity and gastrointestinal tracts, and they have been isolated and implicated in human clinical cases, some leading to deaths. The detection of E. brevis (Table 2 and Figure 2), an environmental inhabitant occasionally found in clinical specimens, underlines the potential threat of obscure pathogens on humans, especially so when the pathogen is adept at surviving in the hospital environment (Sharma et al., 2016). Although there is still a lack of conclusive evidence to resolve the controversial role of head lice as disease vectors, we found substantial genomic evidence pointing to the existence of a large pathogenic microbial community within the head lice. The possibility of head lice transmitting pathogenic bacteria originating from a contaminated blood meal (Boutellis et al., 2013) can prove to be a challenging public health issue.

Despite the thorough surface sterilization process being carried out, diverse numbers of bacteria were found on the surface of head lice samples, that either originated from the environment or as part of the mammalian skin flora. This similar process had been previously adopted (Carpi et al., 2011; Menchaca et al., 2013; Khoo et al., 2016), describing the presence of bacterial communities on the exoskeletons. Therefore, the washing step is deemed necessary to eliminate contaminants from the surroundings (Carpi et al., 2011), with more vigorous procedures incorporated for future studies (Khoo et al., 2016). This is still a limitation as surface sterilization does not remove DNA from the specimen, therefore, normal flora detected in the findings may still be the residual DNA from the host.

The isolation of a relatively small number of head lice from a single site in this study represented another limitation, although interestingly, this could be due to the use of Pongamia pinnata leaf extracts, a traditional medicine of the Orang Asli, to control pediculosis (Anbu et al., 2009). In order to overcome this limitation, more Orang Asli settlements from a wider area can be included, hence, a better representation of the microbial community within the head lice can be obtained. The diversity of bacteria detected in this study showed the propensity of the head lice to act as a host for rare pathogenic bacterial species, although its role as a vector has yet to be demonstrated. Nevertheless, our findings outline the baseline data for the risk and potential hazard of the transmission of infectious diseases from head lice from this community. There is also an urgent need to make an inventory of the list of bacterial communities within the head lice and study its association with pediculosis. In addition, continuous education and awareness programs are vital in improving the hygiene practices among the Orang Asli, crucial in eradicating head lice infestations. 


\section{CONCLUSION}

This study highlights the bacterial diversity in the gut of head lice, with the detection of rare and pathogenic bacteria capable of causing harmful infections. Some of the detected bacterial species have an animal origin, suggesting the potential transfer of zoonotic diseases to the Orang Asli.

\section{Conflict of interest}

The authors declare that they have no conflict of interest.

Acknowledgements. We thank the Department of Orang Asli Development (JAKOA) authorities and village leaders for their cooperation in this study. This work was supported by the University of Malaya (RU005-2017), U.S. Naval Medical Research Center - Asia (Work Unit No. D-1101), the U.S. Department of State, the Ministry of Science Technology and Innovation Malaysia (DSTIN GA017-2016-Subproject 2C), and the Ministry of Education, Malaysia for niche area research under the Higher Institution Centre of Excellence (HiCoE) program (Project MO002-2019).

\section{REFERENCES}

Achermann, Y., Goldstein, E.J., Coenye, T. \& Shirtliff, M.E. (2014). Propionibacterium acnes: from commensal to opportunistic biofilm-associated implant pathogen. Clinical Microbiology Reviews 27(3): 419-440.

Allen, J.M., Reed, D.L., Perotti, M.A. \& Braig, H.R. (2007). Evolutionary relationships of "Candidatus Riesia spp." endosymbiotic Enterobacteriaceae living within hematophagous primate lice. Applied and Environmental Microbiology 73(5): 1659-1664.

Anbu, J.S.J.S., Suraj, R., Rejitha, G., Kalusalingam, A., Vimala, A.G.K.A. \& Hussain, H.A. (2009). In vitro screening of anti-lice activity of Pongamia pinnata leaves. The Korean Journal of Parasitology 47(4): 377-380.
Angelakis, E., Diatta, G., Abdissa, A., Trape, J.-F., Mediannikov, O., Richet, H. \& Raoult, D. (2011a). Altitude-dependent Bartonella Quintana genotype C in head lice, Ethiopia. Emerging Infectious Diseases 17(12): 2357-2359.

Angelakis, E., Rolain, J.M., Raoult, D. \& Brouqui, P. (2011b). Bartonella Quintana in head louse nits. FEMS Immunology and Medical Microbiology 62(2): 244246.

Bachok, N., Nordin, R., Awang, C.W., Ibrahim, N.A. \& Naing, L. (2006). Prevalence and associated factors of head lice infestation among primary schoolchildren in Kelantan, Malaysia. The Southeast Asian Journal of Tropical Medicine and Public Health 37(3): 536-543.

Beims, H., Overmann, A., Fulde, M., Steinert, M. \& Bergmann, S. (2016). Isolation of Staphylococcus sciuri from horse skin infection. Open Veterinary Journal 6(3): 242-246.

Bonilla, D.L., Durden, L.A., Eremeeva, M.E. \& Dasch, G.A. (2013). The biology and taxonomy of head and body lice Implications for louse-borne disease prevention. PLOS Pathogens 9(11): e1003724.

Bonilla, D.L., Kabeya, H., Henn, J., Kramer, V.L. \& Kosoy, M.Y. (2009). Bartonella quintana in body lice and head lice from homeless persons, San Francisco, California, USA. Emerging Infectious Diseases 15(6): 912-915.

Boutellis, A., Mediannikov, O., Bilcha, K.D., Ali, J., Campelo, D., Barker, S.C. \& Raoult, D. (2013). Borrelia recurrentis in head lice, Ethiopia. Emerging Infectious Diseases 19(5): 796-798.

Carpi, G., Cagnacci, F., Wittekindt, N.E., Zhao, F., Ji, Q., Tomsho, L.P., Drautz, D.I., Rizzoli, A. \& Schuster, S.C. (2011). Metagenomic profile of the bacterial communities associated with Ixodes ricinus ticks. PLOS One 6(10): e25604.

Chandren, J.R., Wong, L.P. \& AbuBakar, S. (2015). Practices of dengue fever prevention and the associated factors among the Orang Asli in Peninsular Malaysia. PLOS Neglected Tropical Diseases 9(8): e0003954. 
Chen, B., The, B.-S., Sun, C., Hu, S., Lu, X., Boland, W. \& Shao, Y. (2016). Biodiversity and activity of the gut microbiota across the life history of the insect herbivore Spodoptera littoralis. Scientific Reports 6: 29505.

Chen, Y., Liao, K., Ai, L., Guo, P., Huang, H., Wu, Z. \& Liu, M. (2017). Bacteremia caused by Bergeyella zoohelcum in an infective endocarditis patient: case report and review of literature. $B M C$ Infectious Diseases 17: 271.

Choi, H.S., Bae, E.H., Ma, S.K. \& Kim, S.W. (2017). Peritoneal dialysis-related peritonitis caused by Microbacterium paraoxydans. Japanese Journal of Infectious Diseases 70(2): 195-196.

Delorme, C., Guédon, E., Pons, N., Cruaud, C., Couloux, A., Loux, V., Chiapello, H., Poyart, C., Gautier, C., Sanchez, N., Almeida, M., Kennedy, S.P., Ehrlich, S.D., Gibrat, J.-F., Wincker, P. \& Renault, P. (2011). Complete genome sequence of the clinical Streptococcus salivarius strain CCHSS3. Journal of Bacteriology 193(18): 5041-5042.

Diamoande, S.E., Nguyen-The, C., Guinebretiere, M.-H., Broussole, V. \& Brillard, J. (2015). Role of fatty acids in Bacillus environmental adaptation. Frontiers in Microbiology 6: 813.

Dzeing-Ella, A., Szwebel, T.A., Loubinoux, J., Coignard, S., Bouvet, A., Le Jeunne, C. \& Aslangul, E. (2009). Infective endocarditis due to Citrobacter koseri in an immunocompetent adult. Journal of Clinical Microbiology 47(12): 41854186.

Erdem, I., Dogru, M., Emir, S., Ali, R.K., Elbasan, S., Mutlu, R., Gunes, H. \& Topkaya, A.E. (2015). Unusual presentation of Streptococcus anginosus with severe sepsis, liver abscess secondary to biliary tract perforation. Journal of Medical Cases 6(11): 508-511.

Falagas, M.E., Mathaiou, D.K., Rafailidis, P.I., Panos, G. \& Pappas, G. (2008). Worldwide prevalence of head lice. Emerging Infectious Diseases 14(9): 1493-1494.
Feldmeier, H. (2014). Treatment of pediculosis capitis: a critical appraisal of the current literature. American Journal of Clinical Dermatology 15(5): 401-412.

Frankowski, B.L., Weiner, L.B., Committee on School Health \& Committee on Infectious Diseases. (2002). Head lice. Pediatrics 110(3): 638-643.

Gallego, V., García, M.T. \& Ventosa, A. (2005). Methylobacterium variabile sp. nov., a methylotrophic bacterium isolated from an aquatic environment. The International Journal of Systematic and Evolutionary Microbiology 55(4): 1429-1433.

Khoo, J.-J., Chen, F., Kho, K.L., Ahmad Shanizza, A.I., Lim, F.-S., Tan, K.-K., Chang, L.-Y. \& AbuBakar, S. (2016). Bacterial community in Haemaphysalis ticks of domesticated animals from the Orang Asli communities in Malaysia. Ticks and Tick-borne Diseases 7(5): 929-937.

Khor, C.-S., Mohd-Rahim, N.-F., Hassan, H., Chandren, J.R., Nore, S.-S., Johari, J., Loong, S.-K., Abd-Jamil, J., Khoo, J.-J., Lee, H.-Y., Pike, B.L., Wong, L.P., Lim, Y.A.L., Zandi, K. \& AbuBakar, S. (2018). Seroprevalence of $\mathrm{Q}$ fever among the indigenous people (Orang Asli) of Peninsular Malaysia. Vector-Borne and Zoonotic Diseases 18(3): 131-137.

Kierzkowska, M., Majewska, A., SzymanekMajchrzak, K., Sawicka-Grzelak, A., Mlynarczyk, A. \& Mlynarczyk, G. (2017). In vitro effect of clindamycin against Bacteroides and Parabacteroides isolates in Poland. Journal of Global Antimicrobial Resistance 13: 49-52.

Lesshafft, H., Baier, A., Guerra, H., Terashima, A. \& Feldmeier, H. (2013). Prevalence and risk factors associated with pediculosis capitis in an impoverished urban community in Lima, Peru. Journal of Global Infectious Diseases 5(4): 138-143. 
Leys, N.M.E.J., Ryngaert, A., Bastiaens, L., Verstraete, W., Top, E.M. \& Springael, D. (2004). Occurrence and phylogenetic diversity of Sphingomonas strains in soils contaminated with polycyclic aromatic hydrocarbons. Applied and Environmental Microbiology 70(4): 1944-1955.

Lim, F.-S., Khoo, J.-J., Chen, F., Bell-Sakyi, L., Khor, C.-S., Chang, L.Y. \& Abu Bakar, S. (2017). Initiation of primary cell cultures from embryonic Haemaphysalis bispinosa ticks. Systematic and Applied Acarology 22(3): 323-332.

Mana, N., Louni, M., Parola, P. \& Bitam, I. (2017). Human head lice and pubic lice reveal the presence of several Acinetobacter species in Algiers, Algeria. Comparative Immunology, Microbiology \& Infectious Diseases 53: 3339.

Masron, T., Masami, F. \& Ismail, N. (2013). Orang Asli in Peninsular Malaysia: Population, spatial distribution and socio-economic condition. Journal of Ritsumeikan Social Sciences and Humanities 6: 75-115.

Meister, L. \& Ochsendorf, F. (2016). Head lice - epidemiology, biology, diagnosis and treatment. Deutsches Arzteblatt International 113: 763-772.

Menchaca, A.C., Visi, D.K., Strey, O.F., Teel, P.D., Kalinowski, K., Allen, M.S. \& Williamson, P.C. (2013). Preliminary assessment of microbiome changes following blood-feeding and survivorship in the Amblyomma americanum nymph-to-adult transition using semiconductor sequencing. PloS ONE 8(6): e67129.

Monge, S., Mollema, L., de Melker, H., Sanders, E., van der Ende, A. \& Knol, M. (2018). Clinical characterization of invasive disease caused by Haemophilus influenzae serotype B in a high vaccination coverage setting. Journal of the Pediatric Infectious Diseases Society 8(3): 261-264.
Muhammad Zayyid, M., Saidatul Saadah, R., Adil, A.R., Rohela, M. \& Jamaiah, I. (2010). Prevalence of scabies and head lice among children in a welfare home in Pulau Pinang, Malaysia. Tropical Biomedicine 27(3): 442-446.

Mumcuoglu, K.Y. (2008). Human lice: Pediculus and Pthirus. In: Paleomicrobiology: Past Human Infections, Raoult, D. \& Drancourt, M. (editors) Springer, Berlin, Heidelberg, pp. 215222.

Perotti, M.A., Catala, S.S., Ormeno, A.d.V., Zelazowska, M., Bilinski, S.M. \& Braig, H.R. (2004). The sex ratio distortion in the human head louse is conserved over time. BMC Genetics 5: 10.

Rodríguez, J., Pavía, P., Montilla, M. \& Puerta, C.J. (2011). Identifying triatomine symbiont Rhodococcus rhodnii as intestinal bacteria from Rhodnius ecuadoriensis (Hemiptera: Reduviidae) laboratory insects. International Journal of Tropical Insect Science 31(1-2): 34-37.

Saghafipour, A., Nejati, J., Zahraei-Ramazani, A., Vatandoost, H., Mozaffari, E. \& Rezaei, F. (2017). Prevalence and risk factors associated with head louse (Pediculus humanus capitis) in Central Iran. International Journal of Pediatrics 5(7): 5245-5254.

Sam, I.C., Kahar-Bador, M., Chan, Y.F., Loong, S.K. \& Mohd Nor Ghazali, E. (2008). Multisensitive communityacquired methicillin-resistant Staphylococcus aureus infections in Malaysia. Diagnostic Microbiology and Infectious Diseases 62(4): 437-439.

Sasaki, T., Poudel, S.K., Isawa, H., Hayashi, T., Seki, N., Tomita, T., Sawabe, K. \& Kobayashi, M. (2006). First molecular evidence of Bartonella Quintana in Pediculus humanus capitis (Phthiraptera: Pediculidae), collected from Nepalese children. Journal of Medical Entomology 43(1): 110-112. 
Schrottner, P., Gunzer, F., Schuppel, J. \& Rudolph, W.W. (2016). Identification of Rare Bacterial pathogens by $16 \mathrm{~S}$ rRNA gene sequencing and MALDI-TOF MS. Journal of Visualized Experiments 113: e53176.

Sharma, D., Patel, A., Soni, P., Sharma, P. \& Gupta, B. (2016). Empedobacter brevis meningitis in a neonate: a very rare case of neonatal meningitis and literature review. Case Reports in Pediatrics 2016: 7609602.

Sinniah, B., Sinniah, D. \& Rajeswari, B. (1981). Epidemiology of Pediculus humanus capitis infestation in Malaysian school children. The American Journal of Tropical Medicine and Hygiene 30(3): 734-738.

Šochová, E., Husník, F., Nováková, E., Halajian, A. \& Hypsa, V. (2017). Arsenophonus and Sodalis replacements shape evolution of symbiosis in louse flies. PeerJ 5: e4099.

Soe, M.M., Ismail, S., Sugathan, S., Singh, D., Sinniah, B. \& Hassan, A.K.R. (2018). Prevalence of ectoparasitic infestations: head lice and scabies among Orang Asli school. Malaysian Journal of Public Health Medicine 18(Suppl 1): 59.
Stelzmueller, I., Biebl, M., Wiesmayr, S. Eller, M., Hoeller, E., Fille, M., Weiss, G., Lass-Floerl, C. \& Bonatti, H. (2006). Ralstonia pickettii-innocent bystander or a potential threat? Clinical Microbiology and Infection 12(22): 99-101.

Suzuki, K., Hirai, Y., Morita, F., Nakamura, A., Uehara, Y. \& Naito, T. (2017). Streptobacillus moniliformis bacteremia in a pet shop employee: case report and literature review. Open Forum Infectious Diseases 4(2): ofx038.

Vythilingam, I. et al. (1998). Prevalence of head lice infestation among Orang Asli in Pos Betau using permethrinimpregnated bednets. Malaysian Journal of Medical Laboratory Sciences 10(2): 51-53.

Wong, S.L., Soosai, P., Teoh, Y.L., Han, H.H., Lefevre, I. \& Bock, H.L. (2008). Four is better than nine. A combined diphtheria-tetanus-pertussis-hepatitis B-Haemophilus influenzae type b vaccine for routine immunization in Malaysia. The Southeast Asian Journal of Tropical Medicine and Public Health 39(3): 474-483. 